\title{
Changes in alpha-amylase activity, concentration and isoforms in pigs after an experimental acute stress model: an exploratory study
}

\author{
María Dolores Contreras-Aguilar ${ }^{1}$, Damián Escribano², Silvia Martínez-Subiela', Silvia Martínez-Miró,
} Jose Joaquín Cerón ${ }^{1}$ and Fernando Tecles ${ }^{1 *}$ (i)

\begin{abstract}
Background: Salivary alpha-amylase (SAA) is considered a non-invasive biomarker of acute stress that can be evaluated by changes in activity and concentration, and also by changes in its isoforms, although this last way of evaluation has never been used in veterinary medicine. This research evaluated the changes of sAA by three different ways in which sAA can be evaluated in an experimental acute stress model in six pigs based in a technique of temporarily restraining. These ways of evaluation were 1) activity by a spectrophotometric assay, 2) concentration by a fluorometric assay, and 3) isoforms of the enzyme by a Western blot.

Results: Although salivary cortisol significantly increased due to the stimulus of stress and all the pigs manifested signs of stress by high-pitched vocalization, sAA activity showed an increase of different degree in the six pigs after the stress stimulus, while sAA concentration showed decreases in four of the six pigs. sAA activity did not correlate with SAA concentration or salivary cortisol, and a low correlation was observed between SAA concentration and salivary cortisol $(r=0.48, p=0.003)$. The inter-individual variability was higher in sAA activity than in sAA concentration and salivary cortisol. Finally, three possible isoforms of sAA at 154-160 kDa, 65-66 kDa and 59-60 kDa were observed that showed different dynamics after the stress induction.

Conclusions: Although this pilot study's results should be taken with caution due to the low sample size, it reveals a different behavior between SAA activity and concentration in pig after an acute stressful stimulus leading to evident external signs of stress by high-pitched vocalization, and opens a new field for the evaluation of possible selected isoforms of sAA as potential biomarkers of stress.
\end{abstract}

Keywords: Biomarkers, Isoforms, Salivary alpha-amylase, Stress, Swine

\section{Background}

Nowadays there is an interest in improving the stress evaluation in highly productive indoor-housed pigs since stress can induce behavioral, physiological and immunological alterations leading to a reduction of animal welfare and health [1]. The stress can be evaluated by direct behavioral observations, for example using behavior scoring systems [2-4] or by recording high-pitched

\footnotetext{
* Correspondence: ftecles@um.es

${ }^{1}$ Interdisciplinary Laboratory of Clinical Analysis (Interlab-UMU), Veterinary

School, Campus of Excellence Mare Nostrum, University of Murcia, 30100,

Espinardo, Murcia, Spain

Full list of author information is available at the end of the article
}

vocalizations [5, 6]. However, it can be also objectively assessed by biomarkers that can reflect the pathophysiological responses to stress [7]. Currently there is a growing interest in the use of saliva as a sample for measurement of stress biomarkers [8-11] because saliva is easily obtained and non-invasively compared to blood [12]. Salivary alpha-amylase (sAA) has been proposed as a biomarker for assessing acute psychological stress in humans due to sympathetic activation $[13,14]$. In addition, sAA has been also measured in pig as a sympathetic nervous system (SNS) marker [15], whereas cortisol has been measured to assess activation

(c) The Author(s). 2018 Open Access This article is distributed under the terms of the Creative Commons Attribution 4.0 International License (http://creativecommons.org/licenses/by/4.0/), which permits unrestricted use, distribution, and reproduction in any medium, provided you give appropriate credit to the original author(s) and the source, provide a link to the Creative Commons license, and indicate if changes were made. The Creative Commons Public Domain Dedication waiver (http://creativecommons.org/publicdomain/zero/1.0/) applies to the data made available in this article, unless otherwise stated. 
of hypothalamic-pituitary-adrenocortical (HPA) axis $[7,16]$. In addition to pigs, sAA has been studied in other animal species such as bonobos and other simians [17], horses [10], sheep [18] and dogs [19].

sAA can be evaluated by its enzymatic activity, although a high inter-individual variability in response to stress has been reported in pigs [15], as well in humans [13] and horses [10]. Quantification is another way to evaluate sAA, showing a lower inter-individual variability, as described in horses [10] and humans [20]. Lastly, sAA isoforms and its possible changes after a sAA stimulation have been evaluated in humans $[21,22]$. Despite the interest and use of this enzyme, to the authors' knowledge there are no studies comparing the different ways in which the sAA response against a stressor could be assessed: activity, concentration and changes in isoforms.

The aim of this research was to evaluate the individual changes of sAA in an experimental acute stress model in pig, consisting of a nasal snare immobilization [23], by the three different ways in which sAA can be evaluated: (1) measurement of activity with a spectrophotometric assay, (2) measurement of concentration with a time-resolved immunofluorometric assay (TR-IFMA), and (3) the study of the possible changes in isoforms by western blot (WB). In addition, the sAA results were compared to those obtained with salivary cortisol.

\section{Methods}

\section{Animals and experimental acute stress model}

For this study, male crossbreed pigs (Duroc $\times$ (Landrace $\times$ Large White) in the mid-fattening period (mean age $=$ $104.8 \pm 10.0$ days, mean body weight $=78.3 \pm 6.3 \mathrm{Kg}$ ) from the high sanitary/health-status farm unit of the University of Murcia (Spain) were used. All animals were subjected to a clinical examination prior to the study and no clinical signs of disease were detected. The pigs had access to a nutritionally balanced diet (commercial dry diets based in a corn-soybean meal with $15.5 \%$ of crude protein -CP-; 0.79\% Ileal digestible Lys, \%; and 13.5 MJ of metabolizable energy per $\mathrm{kg}-\mathrm{ME} / \mathrm{kg}-$ ) and water ad libitum (from nipple drinkers) under general commercial housing and husbandry conditions conforming to the European Union Guidelines (Directive 2010/63/EU1). Each pen had an area of $1.139 \mathrm{~m}^{2}$ per animal, being in concordance to the legislation (Council Directive 2001/88/CE). The temperature in the pens was kept between a minimum of $18.0^{\circ} \mathrm{C}$ and a maximum of $19.5{ }^{\circ} \mathrm{C}$. The Murcia University Ethics Committee with the number CEEA 288/2017 approved this study.

The stressful situation consisted of a technique of temporarily restraining pigs commonly used in veterinary practice which has been demonstrated to produce a high stress to pigs [7, 23]. Six pigs were subjected to an immobilization for at least $1 \mathrm{~min}$ with a nasal snare or loop following the procedure described in the literature [15, 23]. Salivary samples were taken the day before the experiment took place, which was considered as basal time (Tb). On the other hand, the same day of the experiment samples were taken $5 \mathrm{~min}$ before restrain (T-5), for the first $30 \mathrm{~s}$ while the nasal snare immobilization was performed $(\mathrm{T}+0 \mathrm{~A})$, for the second $30 \mathrm{~s}$ during immobilization $(\mathrm{T}+0 \mathrm{~B})$, just after immobilization $(\mathrm{T}+0 \mathrm{C})$ and $15 \mathrm{~min}$ later $(T+15)$. The saliva collection at each time lasted 1 min, except for $\mathrm{T}+0 \mathrm{~A}$ and $\mathrm{T}+\mathrm{OB}$. The sampling times were selected to evaluate the possible short-term changes in sAA. The observer responsible for sampling individually recorded if high-pitched vocalizations were present or absent during the restraint period. All animal were sampled between 9:00 to 12:00 a.m. to avoid circadian influences $[24,25]$, by the same person, when animals were in repose, avoiding the race and from pigs housed at different rooms to avoid suggestion; in order to homogenize the experimental conditions for all animals.

\section{Salivary sampling}

Saliva samples were collected by introducing a small sponge around the mouth and it was placed in a collection device (Salivette, Sarstedt, Aktiengesellschaft \& Co, Nümbrecht, Germany), as reported before [23]. Samples were then stored in ice until arrival at the processing laboratory, where devices were centrifuged at $3.000 \times g$ for $10 \mathrm{~min}$ at $4{ }^{\circ} \mathrm{C}$. Saliva was transferred into $1.5 \mathrm{~mL}$ tubes and stored at $-80^{\circ} \mathrm{C}$ until analysis.

\section{Alpha-amylase enzymatic assay}

sAA activity was measured in an automatic analyzer (Olympus UA600, Olympus Diagnostica $\mathrm{GmbH}$ ) by a colorimetric commercial kit (Alpha-Amylase, Beckman Coulter Inc.) following the International Medicine (IFCC) method [26]. This spectrophotometric assay uses 4,6-ethylidene(G7)-pnitrophenol(G1)-alpha-D- maltoheptaoside (G7PNP) as a substrate of the enzyme. The intermediate product of substrate hydrolysis reacts with a-glucosidase, giving p-nitrophenol as the final product of the reaction. The rate of $\mathrm{p}$-nitrophenol formation was directly proportional to the alpha-amylase activity of the sample and was determined by measuring the absorbance at $405 \mathrm{~nm}$. This kit was previously validated for pigs by Fuentes et al. [15], with a intra- and inter-assay coefficients of variation $(\mathrm{CV})$ lower than $10 \%$, and a limit of detection of $11.65 \mathrm{U} / \mathrm{L}$.

\section{Time-resolved immunofluorometric assay}

sAA concentration was measured by a time-resolved immunofluorometric assay (TR-IFMA). The assay consisted of a non-competitive indirect sandwich method based on anti-human sAA polyclonal antibody biotin-labeled as a capture reagent and the anti-human-sAA polyclonal 
antibody $\mathrm{Eu}^{3+}$-chelates labeled as a detector. Streptavidin-coated plates (Streptavidin Microtitration Strips, DELFIA, PerkinElmer, Turku, Finland) were used for the development of this assay. The anti-human sAA polyclonal antibody used was produced by the authors according to standard protocols [27] and using as immunogen human sAA purified in the researchers' laboratory according to the procedures of Peng et al. [28].

The fluorometric assay was validated prior to use for sample measurement. Three saliva samples with a low, medium and high sAA mean concentration (10.7, 30.0 and $65.2 \mathrm{ng} / \mathrm{mL}$, respectively) were measured five times in a single run and in five different days for the determination of intra- and inter-assay precision, respectively. The mean CV was $6.8 \% \pm 0.8 \%$ for intra-assay, and $7.4 \% \pm 2.3 \%$ for inter-assay. Linearity under dilution of two saliva samples yielded Pearson correlation coefficients of $r=0.989 \pm 0.006$ (mean \pm SD). The limit of detection and the lower limit of quantification calculated were $1.4 \mathrm{ng} / \mathrm{mL}$ and $18.14 \mathrm{ng} / \mathrm{mL}$, respectively.

\section{Western blot}

WB was performed in the saliva collected at $\mathrm{Tb}, \mathrm{T}+0 \mathrm{~A}$, $\mathrm{T}+\mathrm{OB}$ and $\mathrm{T}+0 \mathrm{C}$ of three pigs that showed different sAA activity response: pig 1 that showed the highest difference of sAA activity levels, pig 3 that showed intermediate differences and pig 5 that showed the lowest difference in sAA activity along the times. Saliva from pigs diluted to obtain an amount of $1 \mathrm{ng}$ of sAA and $10 \mu \mathrm{g}$ of purified human sAA (ab 77875, Abcam, Cambridge, UK) were used for this approach. Proteins were separated in mini one-dimensional sodium dodecyl sulphate polyacrylamide gels (SDS-PAGE) containing $0.1 \%(w / v)$, with a separating gel prepared in $12 \%(\mathrm{w} / \mathrm{v})$ and a stacker gel prepared in $4 \%(\mathrm{w} / \mathrm{v})$ according to the methodology described by Laemmli [29]. Then, the proteins in SDS-PAGE were transferred to nitrocellulose membranes (Bio-Rad Laboratories Inc., Hercules, CA, USA). The same rabbit polyclonal antibody against human sAA used in the TR-IFMA (1:8000 dilution) was employed as primary antibody, while anti-rabbit IgG horseradish peroxidase (HRP)-conjugated goat polyclonal antibody (ab 6721, Abcam, Cambrigde, UK) (1:12000) was used as secondary antibody, and signal was detected using Pierce ECL2 kit (Pierce, Thermo Fisher Scientific, USA) and ImageQuant ${ }^{\mathrm{TM}}$ scanner (GE Healthcare, Uppsala, Sweden). The average intensity of each band, defined as the volume of the band feature divided by its area, was analyzed by using ImageQuant $^{\mathrm{Tm}}$ TL 8.1 (GE Healthcare, Uppsala, Sweden).

\section{Cortisol measurement}

Salivary cortisol was evaluated also for comparative purposes [30] with an automated chemiluminescent immunoassay (Immulite 1000 cortisol, Siemens Medical Solutions Diagnostics) validated for pigs' saliva [8]. The intra- and inter-assay CV was lower than $16 \%$, and a limit of detection of $0.016 \mu \mathrm{g} / \mathrm{dL}$.

\section{Statistical methods}

Arithmetic means, standard deviations (SD), CV and linear regression analyses were calculated using routine descriptive statistical procedures by spreadsheet (Excel 2000, Microsoft Corporation, Redmond, Washington, USA). To determine if sAA and salivary cortisol values obtained at different times in the stressful situation were statistically different, a Friedman test and a Dunn's multiple comparisons test were used. A Spearman correlation test was performed to compare sAA activity, sAA concentration and salivary cortisol results. The inter-individual variability of the sAA values (activity and concentration) and salivary cortisol in each time were expressed as the $\mathrm{CV}$ value, calculated as the SD divided by the mean of the values of the different individuals and then multiplied by 100. These statistical analyses were calculated using Graph Pad Prism 6 (GraphPad Software, San Diego, CA, USA).

\section{Results}

\section{Activity and concentration of alpha-amylase in saliva}

sAA activity (Fig. 1a) showed a significant change between the times $\left(\chi^{2}=12.76, \mathrm{df}=5, P=0.026\right)$, but the multiple comparisons test did not show any significant increase at any time $(P>0.08$ for all times compared). sAA concentration (Fig. 1b) did not show significant changes along the times $\left(\chi^{2}=7.33, \mathrm{df}=5, P=0.197\right)$. Salivary cortisol significantly changed between the times $\left(\chi^{2}=13.71, \mathrm{df}=5\right.$, $P=0.033)$, with an increase $(P=0.045)$ at $\mathrm{T}+0 \mathrm{~B}$ compared to TB (Fig. 1c). There was a significant correlation between sAA concentration and salivary cortisol $(n=36, r=0.48,95 \%$ confidence interval [CI] $0.18-0.71, P=0.003)$. However, no correlation was observed between sAA activity and sAA concentration $(n=36, \quad r=0.16, \quad 95 \% \quad \mathrm{CI}-0.18-0.47, \quad P=0.338)$, and between sAA activity and salivary cortisol $(n=36, r=0.14$, 95\% CI $-0.21-0.45, P=0.418)$. Recurrent high-pitched vocalizations were observed in the six pigs during the restraint period.

When sAA activity values were individually evaluated (Fig. 2a), the differences of sAA activity between the times had an average value higher than $1000 \mathrm{U} / \mathrm{L}$ in pigs $1(4166.4 \mathrm{U} / \mathrm{L})$ and $6(1225.5 \mathrm{U} / \mathrm{L})$; higher than $500 \mathrm{U} / \mathrm{L}$ in pigs $2(743.6 \mathrm{U} / \mathrm{L}), 3(826.2 \mathrm{U} / \mathrm{L})$ and 4 $(548.1 \mathrm{U} / \mathrm{L})$; and lower than $100 \mathrm{U} / \mathrm{L}$ in pig $5(71.1 \mathrm{U} / \mathrm{L})$. Peaks of activity were observed in pig 1 at $\mathrm{T}+0 \mathrm{~A}$, in pig 2 at $\mathrm{T}+0 \mathrm{C}$ and in pigs 3 and 6 at $\mathrm{T}+0 \mathrm{~B}$. In pig 4 , the peak was observed at T-5.

When sAA concentration was individually evaluated between times (Fig. 2b), a decrease was observed at $\mathrm{T}+0 \mathrm{C}$ 

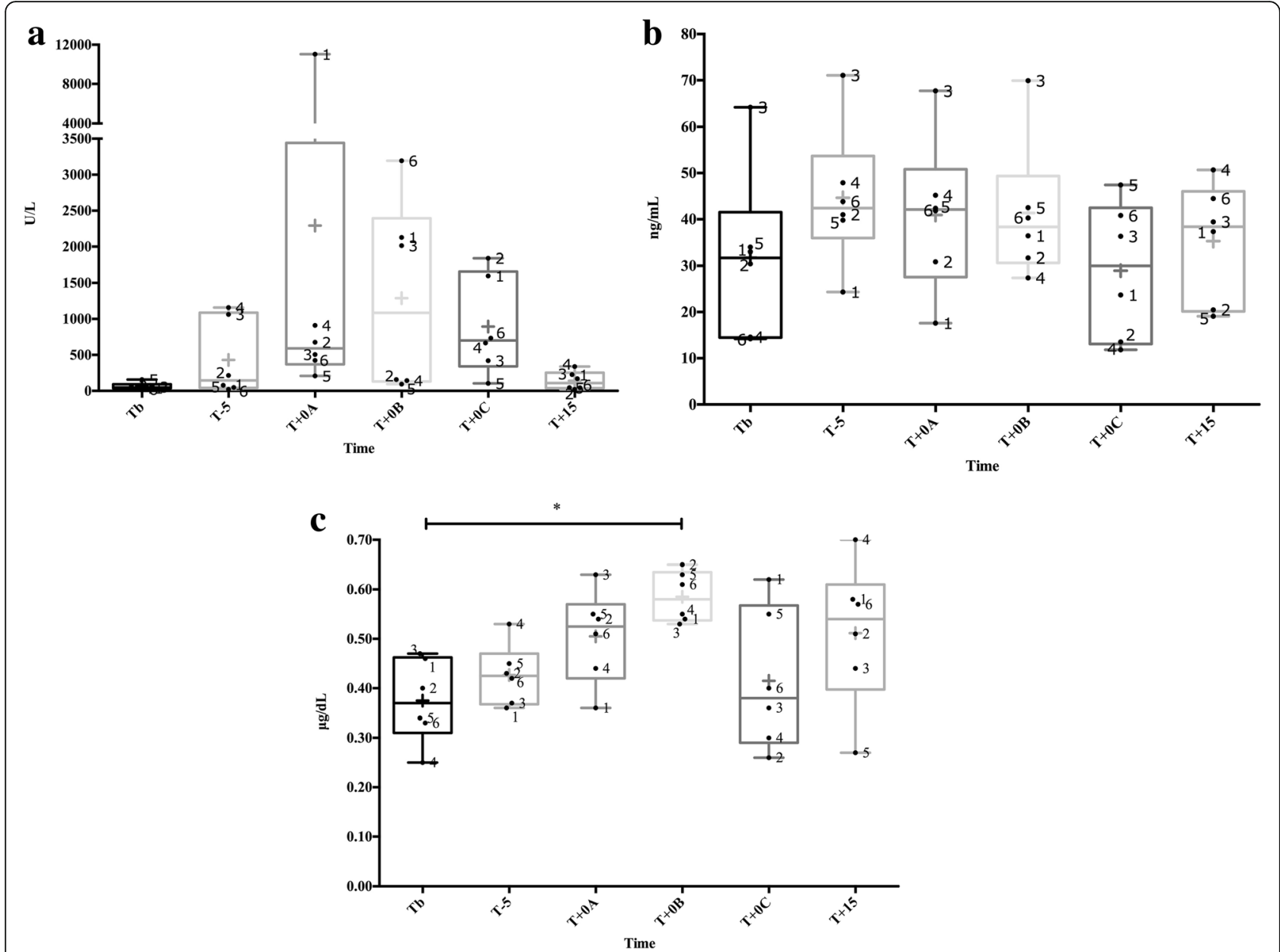

Fig. 1 Salivary alpha-amylase (SAA) activity (a), concentration (b) and salivary cortisol (c) during a nasal snare immobilization for 1 min from six pigs. The times were the day before at basal time (Tb), 5 min before (T-5), the firsts $30 \mathrm{~s}$ while the nasal snare immobilization was being performed $(T+O A)$, the seconds $30 \mathrm{~s}$ while the nasal snare immobilization was being performed $(T+O B)$, just after the immobilization $(T+0 C)$ and $15(T+15)$ minutes later. The plots show medians (line within box), mean (cross inside the box), 25th and 75th percentiles (boxes), min and max values (whiskers) and individual values (points). The numbers mean the name of the pigs. Asterisks indicate significant post-hoc difference (Dunn's multiple comparisons test): * $P<0.05$

in all pigs, with the exception of the pig 5 and 6 . The inter-individual mean variability (Table 1 ) of the sAA activity $(109.2 \% \pm 42.2)$ was higher $(2.5$-fold and 5.1 -fold, respectively) than in sAA concentration $(42.7 \% \pm 9.5)$ and salivary cortisol $(21.2 \% \pm 9.3)$.

\section{Changes in isoforms patterns}

WB results are presented in Fig. 3. Pigs saliva showed a band of $59-60 \mathrm{kDa}$ in all times. On the other hand, pig 5 showed a band with 154-160 kDa along the different times. Additionally, a band at $65-66 \mathrm{kDa}$ was observed in pigs 1 and 3 in the stress times only when sAA activity values were higher than $1000 \mathrm{U} / \mathrm{L}$. The bands with 59-60 kDa and 154-160 kDa were also marked in the commercial human purified sAA (Additional file 1).

\section{Discussion}

In this paper, changes in sAA activity, concentration, and its possible isoforms were evaluated after an experimental induced stress in pigs. The increase of salivary cortisol, considered as a biomarker of stress [7], that appeared in all pigs used in our study after the restraint, would indicate that our experimental procedure was able to generate a stress in the animals. In addition, the high-pitched vocalizations emitted by the six pigs during the restrain period could indicate a negative emotional state [5]. sAA activity showed peaks of increase in four of the six pigs after the stressor at different times and with different intensity, however sAA concentration decreased in many animals after the stimulus. A reason for this different behavior could be the fact that activity and concentration are associated to different possible isoforms of sAA. 


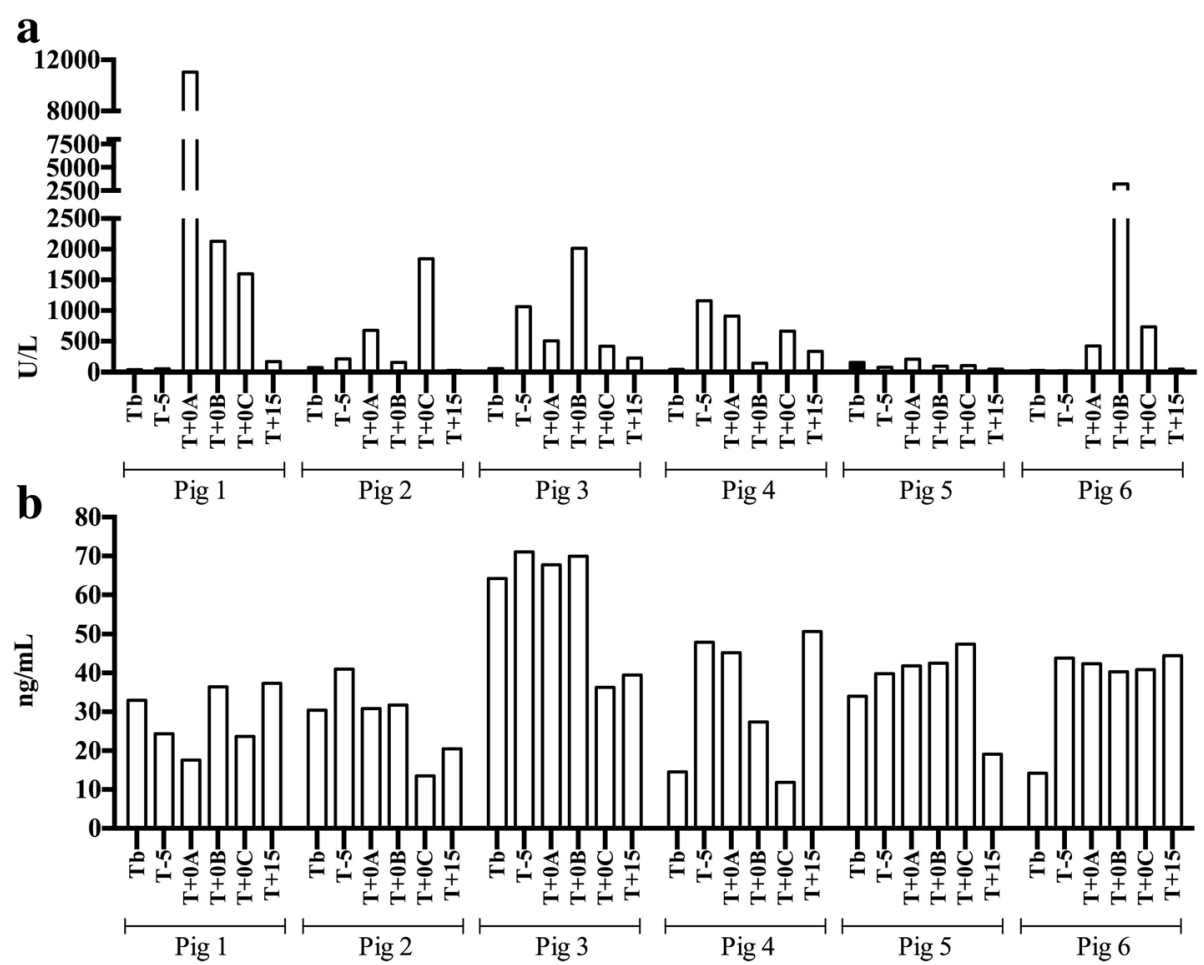

Fig. 2 Individual levels of salivary alpha-amylase (SAA) activity (a) and concentration (b) obtained from six pigs before and after nasal snare immobilization for $1 \mathrm{~min}$. The times were the day before at basal time (Tb), $5 \mathrm{~min}$ before (T-5), the firsts $30 \mathrm{~s}$ while the nasal snare immobilization was being performed $(T+O A)$, the seconds $30 \mathrm{~s}$ while the nasal snare immobilization was being performed $(T+0 B)$, just after the immobilization $(T+0 C)$ and $15(T+15)$ minutes later. Bars show the total value from each individual at each time

For the analysis of sAA isoforms, serial samples from individuals representing different sAA response (one with a major sAA response, one with a moderate sAA response and one with a minor response) were analysed. According to the WB's results, different sAA isoforms appeared depending of the intensity of the sAA response. In humans it has been demonstrated that these sAA isoforms can

Table 1 Inter-individual variability (\%) of salivary alpha-amylase (SAA) measured by enzymatic activity (U/L) and concentration $(\mathrm{ng} / \mathrm{mL})$, and of salivary cortisol concentration $(\mu \mathrm{g} / \mathrm{dL})$ after the experimental nasal snare immobilization for $1 \mathrm{~min}$ in 6 pigs

\begin{tabular}{llll}
\hline \multicolumn{3}{l}{ sAA interindividual variability (\%) } & \\
\hline & SAA & salivary cortisol \\
\cline { 2 - 3 } & $\mathrm{U} / \mathrm{L}$ & $\mathrm{ng} / \mathrm{mL}$ & $\mu \mathrm{g} / \mathrm{dL}$ \\
\hline $\mathrm{Tb}$ & 75.3 & 57.51 & 22.5 \\
$\mathrm{~T}-5$ & 123.6 & 34.09 & 14.42 \\
$\mathrm{~T}+$ OA & 187.0 & 40.64 & 18.61 \\
$\mathrm{~T}+$ OB & 103.5 & 36.33 & 8.77 \\
$\mathrm{~T}+$ OC & 76.2 & 51.16 & 34.2 \\
$\mathrm{~T}+15$ & 89.4 & 36.43 & 28.6 \\
Mean & 109.2 & 42.7 & 21.2 \\
$\mathrm{SD}^{\mathrm{a}}$ & 42.2 & 9.5 & 9.3
\end{tabular}

${ }^{\mathrm{a}} \mathrm{SD}$ standard deviation change after sAA stimulation [22] and changes observed in these isoforms could influence the concentration and activity of the enzyme. However, as well as occurs with activity, there was a high inter-individual variability in the response of the different isoforms. The bands with 59$60 \mathrm{kDa}$ and $154-160 \mathrm{kDa}$ observed in the WB from porcine saliva had the same molecular weight than bands marked in the commercial purified sAA from humans (Additional file 1). In addition, the band with 59-60 kDa could correspond to the glycosylated isoform described in humans [21]. So it could be postulated that the changes observed in the bands in the WB study could be due to different amylase isoforms. This could open a new line for the evaluation of possible selected isoforms of sAA as potential biomarkers of stress, since for example the isoform with a molecular weight of $65-66 \mathrm{kDa}$ could be related with major response in sAA activity after an stress, since appeared in pigs showing the higher sAA activity response to a stressful stimulus, whereas the isoform of 154-160 kDa could be related with minor response in sAA activity after a stress condition.

A lack of correlation between sAA activity and concentration was observed in our study. Divergences between activity and concentration values have been described for other enzymes such as Paraoxonase-1 (PON1) in human 


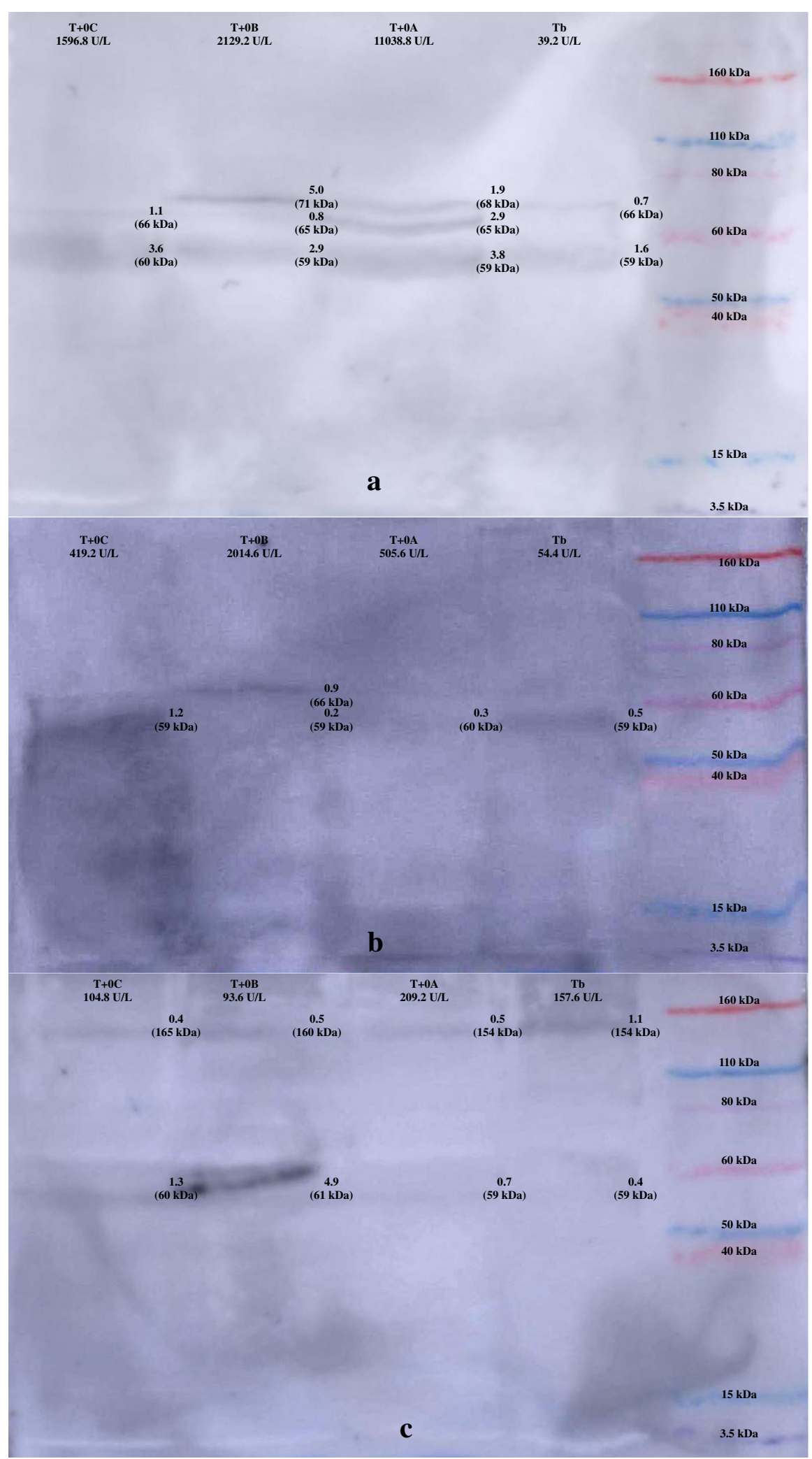

Fig. 3 (See legend on next page.) 
(See figure on previous page.)

Fig. 3 Western blot from pig saliva in an experimental nasal snare immobilization. Saliva samples from Pig 1 (a), Pig 3 (b) and Pig 5 (c) with one ng per well of salivary alpha-amylase ( $\mathrm{SAA}$ ) at the day before at basal time (Tb), the firsts $30 \mathrm{~s}$ while the nasal snare immobilization was being performed $(T+O A)$, the seconds $30 \mathrm{~s}$ while the nasal snare immobilization was being performed $(T+O B)$ and just after the immobilization $(T+0 C)$. Molecular weight markers (Novex Sharp Pre-Stained, Invitrogen, Carlsbad, California). Arrows mark positive bands in pig's saliva, and numbers above indicate the average intensity $\left(\times 10^{3}\right)$

serum [31]. In the sAA case, it could be postulated that post-translational changes [21] could modify the enzyme's activity, although the concentration release does not show significant changes. In addition, we found no correlation between sAA activity and salivary cortisol. This is in line with previous reports previously described $[15,32]$ and could be due to the possible activation of the different related stress axis [33, 34] having a different time of response, with the sAA release being faster than the cortisol. Finally, the positive correlation between sAA concentration and salivary cortisol detected in our study should be taken with caution since $r$ values below 0.50 are considered as weak [35], and both did not show a similar tendency along the times.

In this study, sAA activity showed a high inter-individual variability as it has been previously reported in pigs subjected to an acute stress model [15]. This high inter-individual variability was also observed in sAA concentration and it could be the cause for not obtaining significant differences in both sAA activity and concentration after the stress model. Biomarkers assessing of stress usually show inter-individual variability $[8,13,36]$, probably due to the complexity of stress reaction [37] and because the stress response varies according to idiosyncrasy of the individual [38]. However, this inter-individual variability was higher in sAA activity, being in agreement with previous reports $[10,13,20]$. The higher inter-individual variability in SAA activity could be due to the fast-reacting biological response of the enzyme, and there is a large array of factors with potential to stimulate or inhibit amylase activity [13], that could be a potential limitation for its application as a stress biomarker.

It is important to point out that this is an exploratory study and the results observed should be taken as preliminary ones due to the low sample size. Further studies should be performed in large populations and with other different stress conditions in order to corroborate our findings. In addition, it would be of interest the development of studies to clarify the high inter-individual variability in SAA behavior after a stress induction that we found in our experimental conditions.

\section{Conclusion}

This study reveals that sAA activity and concentration behave in a different way in pigs after an experimental acute stress stimulus, showing a high inter-individual variation in their response. In addition, it reports the presence of sAA isoforms in pig saliva that show a different response after a stress. Although these results should be taken as preliminary ones due to the pilot nature of this study with a low sample size, it could open a new line for the evaluation of possible selected isoforms of sAA as potential biomarkers of stress.

\section{Additional file}

Additional file 1: Western blot from purified human salivary alpha-amylase (sAA, ab 77875, Abcam, Cambrigde, UK). Molecular weight markers (Novex Sharp Pre-Stained, Invitrogen, Carlsbad, California). (PDF 16 kb)

\section{Abbreviations \\ Cl: Confidence interval; CP: Crude protein; CV: Coefficient of variation; HPA: Hypothalamic-pituitary-adrenocortical; HRP: Horseradish peroxidase; ME: Metabolizable energy; PON1: Paraoxonase-1; sAA: Salivary apha-amylase; SD: Standard deviation; SDS-PAGE: One-dimensional sodium dodecyl sulphate polyacrylamide gel electrophoresis; SNS: Sympathetic nervous system; TR-IFMA: Time-resolved immunofluorometric assay; WB: Western blot}

\section{Acknowledgements}

This work was supported by the Seneca foundation of Murcia Regional Government, Spain (grant number 19894/GERM/15). D. Escribano was granted by the postdoctoral program 'Juan de la Cierva' of the 'Ministerio de Economía y Competitividad', Spain. M. Contreras-Aguilar was granted by the predoctoral contract 'FPU' of University of Murcia (R- 605/2016), Spain.

\section{Funding}

Seneca Foundation of Murcia Region (grant number: 19894/GERM/15).

\section{Availability of data and materials}

The datasets generated and/or analysed during the current study are not publicly available due legal reasons but are available from the corresponding author on reasonable request.

\section{Authors' contributions}

MDCA and JJC conceived and designed the study. MDCA and DE collected the data. MDCA were involved in the chemical analysis. MDCA, DE, SMS, SMM, JJC and FT were involved in the data interpretation. MDCA drafted the manuscript and DE, SMS, SMM, JJC and FT critically read and edited the manuscript.

\section{Ethics approval and consent to participate}

This project has been approved by the Murcia University Ethics Committee with the number CEEA-OH 235/2018.

\section{Consent for publication}

Not applicable.

\section{Competing interests}

None of the authors has any financial or personal relationships that could inappropriately influence or bias the content of the paper. 


\section{Publisher's Note}

Springer Nature remains neutral with regard to jurisdictional claims in published maps and institutional affiliations.

\section{Author details \\ 'Interdisciplinary Laboratory of Clinical Analysis (Interlab-UMU), Veterinary School, Campus of Excellence Mare Nostrum, University of Murcia, 30100, Espinardo, Murcia, Spain. ${ }^{2}$ Department of Food and Animal Science, School of Veterinary Medicine, Universitat Autònoma de Barcelona, 08193, Bellaterra, Barcelona, Spain. ${ }^{3}$ Department of Animal Production, Veterinary school, Campus of Excellence Mare Nostrum, Murcia, Spain.}

Received: 4 June 2018 Accepted: 17 August 2018 Published online: 29 August 2018

\section{References}

1. Prunier A, Heinonen M, Quesnel H. High physiological demands in intensively raised pigs : impact on health and welfare. Animal. 2010;4:886-98.

2. Smulders $D$, Verbeke $G$, Mormède $P$, Geers R. Validation of a behavioral observation tool to assess pig welfare. Physiol Behav. 2006;89:438-47.

3. Morton D, Griffiths P. Guidelines on the recognition of pain, distress and discomfort in experimental animals and an hypothesis for assessment. Vet Rec. 1985;116:431-6.

4. O'Driscoll K, Teixeira DL, O'Gorman D, Taylor S, Boyle LA. The influence of a magnesium rich marine supplement on behaviour, salivary cortisol levels, and skin lesions in growing pigs exposed to acute stressors. Appl Anim Behav Sci. 2013;145:92-101.

5. Dokmanović M, Velarde A, Tomović V, Glamočlija N, Marković R, Janjić J, et al. The effects of lairage time and handling procedure prior to slaughter on stress and meat quality parameters in pigs. Meat Sci. 2014:98:220-6.

6. Marx G, Horn T, Thielebein J, Knubel B, Von BE. Analysis of pain-related vocalization in young pigs. J Sound Vib. 2003;266:687-98.

7. Martínez-Miró S, Tecles F, Ramón M, Escribano D, Hernández F, Madrid J, et al. Causes, consequences and biomarkers of stress in swine : an update. BMC Vet Res. 2016;12:1-9.

8. Escribano D, Fuentes-Rubio M, Ceron JJ. Validation of an automated chemiluminescent immunoassay for salivary cortisol measurements in pigs. J Vet Diagn Investig. 2012;24:918-23.

9. Escribano D, Gutiérrez AM, Tecles F, Cerón JJ. Changes in saliva biomarkers of stress and immunity in domestic pigs exposed to a psychosocial stressor. Research in veterinary science. Elsevier BV. 2015;102:38-44.

10. Fuentes-Rubio M, Fuentes F, Otal J, Quiles A, Tecles F, Cerón JJ, et al. Measurements of salivary alpha-amylase in horse: comparison of 2 different assays. J Vet Behav: Clin Appl Res. 2015;10:122-7.

11. Lensen CMM, Moons CPH, Diederich C. Saliva sampling in dogs: how to select the most appropriate procedure for your study. J Vet Behav: Clin Appl Res Elsevier Inc. 2015:10:504-12.

12. Tsuchiya K, Saidin MY, Bin, Inoue T, Kajiwara K, Kimura M. Qualitative measurement of pain by analysing the salivary alpha amylase. Precision Eng Elsevier Inc. 2014;38:257-60.

13. Rohleder N, Nater UM. Determinants of salivary a-amylase in humans and methodological considerations. Psychoneuroendocrinology. 2009:34:469-85.

14. Petrakova L, Doering BK, Vits S, Engler H, Rief W, Schedlowski M, et al. Psychosocial stress increases salivary alpha-amylase activity independently from plasma noradrenaline levels. PLoS One. 2015;10:1-9.

15. Fuentes M, Tecles F, Gutierrez A, Otal J, Martinez-Subiela S, Ceron JJ. Validation of an automated method for salivary alpha-amylase measurements in pigs (Sus scrofa domesticus) and its application as a stress biomarker. J Vet Diagn Investig. 2011;23:282-7.

16. Kudielka BM, Schommer NC, Hellhammer DH, Kirschbaum C. Acute HPA axis responses, heart rate, and mood changes to psychosocial stress (TSST) in humans at different times of day. Psychoneuroendocrinology. 2004;29:983-92.

17. Behringer V, Deschner T, Möstl E, Selzer D, Hohmann G. Stress affects salivary alpha-amylase activity in bonobos. Physiol Behav. 2012;105:476-82.

18. Fuentes-Rubio M, Fuentes F, Otal J, Quiles A, Luisa HM. Validation of an assay for quantification of alpha-amylase in saliva of sheep. Can J Vet Res. 2016;80:197-202
19. Contreras-Aguilar MD, Tecles F, Martínez-Subiela S, Escribano D, Bernal LJ, Cerón JJ. Detection and measurement of alpha- amylase in canine saliva and changes after an experimentally induced sympathetic activation. BMC Vet Res. 2017;13:1-6.

20. Contreras-Aguilar MD, Escribano D, Martínez-Subiela S, Martínez-Miró S, Rubio M, Tvarijonaviciute A, et al. Influence of the way of reporting alphaamylase values in saliva in different naturalistic situations : a pilot study. PLoS One. 2017;12:1-13.

21. Hirtz C, Chevalier F, Centeno D, Rofidal V, Egea JC, Rossignol M, et al. MS characterization of multiple forms of alpha-amylase in human saliva. Proteomics. 2005:5:4597-607.

22. Yang Z, Chen L, Zhang M, Lin J, Zhang J, Chen W. Age differences of salivary alpha-amylase levels of basal and acute responses to citric acid stimulation between Chinese children and adults. Front Physiol. 2015;6:1-11.

23. Escribano D, Soler L, Gutiérrez AM, Martínez-Subiela S, Cerón JJ. Measurement of chromogranin a in porcine saliva: validation of a timeresolved immunofluorometric assay and evaluation of its application as a marker of acute stress. Animal. 2013;7:640-7.

24. O'Donnell K, Kammerer M, O'Reilly R, Taylor A, Glover V. Salivary alphaamylase stability, diurnal profile and lack of response to the cold hand test in young women. Stress. 2009;12:549-54.

25. Nater UM, Rohleder N, Schlotz W, Ehlert U, Kirschbaum C. Determinants of the diurnal course of salivary alpha-amylase. Psychoneuroendocrinology. 2007:32:392-401.

26. van der Heiden C, Bais R, Gerhardt W, Lorentz K, Rosalki S. IFCC methods for measurement of catalytic concentration of enzymes - part 9. IFCC method for alpha-amylase \{[1,4-alpha-D-glucan 4-glucanohydrolase, EC 3.2.1.1]. Clin Chim Acta. 1999:281:S5-39.

27. University of California B. Guidelines for the production of antibodies in laboratory animals. In: Animal care and use committee; 2014.

28. Peng Y, Chen X, Sato T, Rankin SA, Tsuji RF, Purification GY. High-resolution top-down mass spectrometric characterization of human salivary alphaamylase. Anal Chem. 2012:84:3339-46.

29. Laemmli UK. Cleavage of structural proteins during assembly of head of bacteriophage-T4. Nature. 1970;227:680

30. Cook NJ, Schaefer AL, Lepage P, Jones SDM. Radioimmunoassay for cortisol in pig saliva and serum. J Agric Food Chem. 1997;45:395-9.

31. Parra S, Alonso-Villaverde C, Coll B, Ferré N, Marsillach J, Aragonès G, et al. Serum paraoxonase-1 activity and concentration are influenced by human immunodeficiency virus infection. Atherosclerosis. 2006;194:175-81.

32. Contreras-Aguilar MD, Escribano D, Martín-cuervo M, Tecles F, Cerón JJ. Salivary alpha-amylase activity and cortisol in horses with acute abdominal disease : a pilot study. BMC Vet Res. 2018;14:1-7.

33. Engert V, Efanov SI, Duchesne A, Vogel S, Corbo V, Pruessner JC. Differentiating anticipatory from reactive cortisol responses to psychosocial stress. Psychoneuroendocrinology. 2013;38:1328-37.

34. Sumter SR, Bokhorst CL, Miers AC, Van Pelt J, Westenberg PM. Age and puberty differences in stress responses during a public speaking task: do adolescents grow more sensitive to social evaluation? Psychoneuroendocrinology. 2010;35:1510-6.

35. Milton JS. Statistical Methods in The biological and health sciences. 3th editio. Journal of chemical information and modeling. Radford, VA, USA: McGraw-HILL; 2013.

36. Lennartsson A. Effects of Psychosocial Stress on DHEA and DHEA-S levels. Acute and long-term effects. University of Gothenburg; 2013.

37. Fazio E, Ferlazzo A. Evaluation of stress during transport. Vet Res Commun. 2003;27:519-24

38. Rushen J. Using aversion learning techniques to assess the mental state, suffering, and welfare of farm animals. J Anim Sci. 1996:74:1990-5. 